MATHEMATICS OF COMPUTATION

Volume 71, Number 240, Pages 1421-1430

S 0025-5718(01)01398-9

Article electronically published on December 5, 2001

\title{
DERIVATIVE SUPERCONVERGENT POINTS IN FINITE ELEMENT SOLUTIONS OF HARMONIC FUNCTIONS- A THEORETICAL JUSTIFICATION
}

\author{
ZHIMIN ZHANG
}

\begin{abstract}
Finite element derivative superconvergent points for harmonic functions under local rectangular mesh are investigated. All superconvergent points for the finite element space of any order that is contained in the tensorproduct space and contains the intermediate family can be predicted. In the case of the serendipity family, results are given for finite element spaces of order below 6 . The results justify the computer findings of Babuška, et al.
\end{abstract}

\section{INTRODUCTION}

Derivative superconvergent points are those special points where the convergent rate of the derivative of the finite element solution exceeds the possible global rate. This phenomenon has been analyzed mathematically because of its practical importance in finite element computations. For literature, the reader is referred to [3], 6]. So far, most superconvergence investigations have concentrated on the second-order elliptic problems, especially the Poisson equation. In 1996, Babuška et al. developed a "computer-based proof" [2] that predicted all superconvergent points not only for the Poisson equation, but also for the Laplace and the linear elasticity equations, on four mesh patterns of triangular elements and on three families of rectangular elements of degree $n, 1 \leq n \leq 7$. The actual superconvergent points were located by a computer algorithm and up to 10 digits were provided in their reported data (see also [1]).

The main assumptions in [2] are (a) there is no roundoff error, (b) the mesh is locally translation-invariant, and (c) the solution is sufficiently smooth locally and the pollution error is under control. The central idea is to majorize the finite element solution error by a polynomial of one degree higher than the finite element space being used. Therefore, the search for superconvergent points is transferred to a search for intersections of some polynomial contours. At this moment, the computer is used to actually locate those intersections.

In an earlier work [7], the author analytically located those intersections which represent superconvergent points for the Poisson equation under local rectangular meshes. The result justified those computer findings in [2].

Received by the editor November 21, 2000.

2000 Mathematics Subject Classification. Primary 65N30.

Key words and phrases. Superconvergence, finite element, harmonic function.

(C)2001 American Mathematical Society 
The current investigation is the continuation of this previous effort. Here the harmonic functions (solutions of the Laplace equation) are of concern. We shall study the derivative superconvergent points for harmonic functions under local rectangular meshes. While results for the tensor-product space and intermediate elements are the same as those for the Poisson equation, superconvergent points are quite different for the serendipity family. In fact, the situation is more interesting and "nontrivial" for the harmonic functions with the serendipity element. It is known from previous results [2], 7] that there are no derivative superconvergent points for the Poisson equation for even order $n=2 k \geq 4$ serendipity elements and there are only three derivative superconvergent points for Poisson equation for odd order $n=2 k+1>3$ serendipity elements. However, there are plenty of derivative superconvergent points for harmonic functions in both even and odd order serendipity elements, which we shall demonstrate in this work. Indeed, derivative superconvergent points are much richer for harmonic functions than for the Poisson equation. Again, theoretical results in the current article justify the computer findings in 2]. Note that in the theoretical analysis there is no need for assumption (a) in the computer-based proof.

We would like to point out that most of the superconvergent points for harmonic functions are not symmetry points in the sense of [5] by Schatz, Sloan, and Wahlbin, and therefore cannot be predicted by the symmetry theory (see also [6]).

\section{ORTHOGONAL DECOMPOSITION OF PERIODIC POLYNOMIALS}

We shall make assumptions (b) and (c) from now on. The basic result from previous works along this line is that the task of finding derivative superconvergent points can be narrowed down to a master cell or equivalently to the reference element $\widehat{K}=[-1,1]^{2}$. This is based on the key observation that when the exact solution is sufficiently smooth and the local mesh is translation invariant, then the finite element approximation local error can be majorized by the approximation error to polynomials of 1 degree higher (than the finite element local space), an error which behaves periodically. Naturally, we need to introduce $P P_{n}(\widehat{K})$, the space of periodic polynomials of degree not greater than $n$ on $\widehat{K}$. That is to say, for any $f \in P_{n}(\widehat{K}), f$ is a polynomial of degree $(\leq n)$ that satisfies $f(x, 1)=f(x,-1)$, $f(1, y)=f(-1, y)$.

The characteristic of the space $P P_{n}(\widehat{K})$ is the following lemma which is proved in 7].

\section{Lemma 2.1.}

$$
P P_{n}(\widehat{K})=\operatorname{Span}\left\{1, \phi_{k}(x), \phi_{k}(y), k=2,3, \ldots, n ; \phi_{i}(x) \phi_{j}(y), i+j \leq n, i, j \geq 2\right\}
$$

with

$$
\phi_{k+1}(x)=\sqrt{\frac{2 k+1}{2}} \int_{-1}^{x} L_{k}(t) d t, \quad k \geq 1,
$$

where $L_{k}$ is the Legendre polynomial of degree $k$ on $[-1,1]$.

Further, we consider the orthogonal decomposition of $P P_{n}(\widehat{K})$ under the Laplace operator. Toward this end, we define

$$
\Psi_{n}(\widehat{K})=\left\{u \in P P_{n}(\widehat{K}) \mid \quad \int_{\widehat{K}} \nabla u \nabla v=0 \quad \forall v \in P P_{n-1}(\widehat{K})\right\} .
$$


Then by the Gram-Schmidt process, we can decompose $P P_{n}(\widehat{K})$ into

$$
P P_{n}(\widehat{K})=P P_{0}(\widehat{K}) \oplus \Psi_{2}(\widehat{K}) \oplus \cdots \oplus \Psi_{n-1}(\widehat{K}) \oplus \Psi_{n}(\widehat{K}) .
$$

Note that $P P_{0}(\widehat{K})=\operatorname{Span}\{1\}$ and $\Psi_{1}(\widehat{K})=\{0\}$.

Let $V_{n}(\widehat{K})$ be the finite element local space on the reference element. Then we have the following result (see [2, 6], and 7] for the proof).

Theorem 2.1. Under assumptions (b) and (c), derivative superconvergent points of $V_{n}(\widehat{K})$ along the $x$-direction for the Poisson equation are the intersections of the contours

$$
\left\{\frac{\partial \psi}{\partial x}=0 \mid \psi \in \Phi_{n+1}(\widehat{K})\right\}
$$

where

$$
\Phi_{n+1}(\widehat{K})=\left\{\psi \in P P_{n+1}(\widehat{K}) \backslash V_{n}(\widehat{K}) \mid \quad \int_{\widehat{K}} \nabla \psi \nabla v=0 \quad \forall v \in V_{n}(\widehat{K})\right\} .
$$

Let $P_{n}(\widehat{K})$ be the space of complete polynomials of degree $n$ on $\widehat{K}$ and let $Q_{n}(\widehat{K})$ be the tensor-product space of order $n$ on $\widehat{K}$. Then

$$
P_{n+1}(\widehat{K}) \cap Q_{n}(\widehat{K})=P_{n+1}(\widehat{K}) \backslash\left\{x^{n+1}, y^{n+1}\right\}
$$

is the intermediate element of degree $n$, and

$$
S_{n}(\widehat{K})=P_{n}(\widehat{K}) \cup\left\{x^{n} y, x y^{n}\right\}
$$

is the serendipity element of degree $n$.

Apply Theorem 2.1 to the case of harmonic functions, and we have

Theorem 2.2. Under assumptions (b) and (c), assume further that the finite element local space includes the serendipity family, i.e., $S_{n}(\widehat{K}) \subset V_{n}(\widehat{K})$. Then, derivative superconvergent points (along the $x$-direction) of harmonic functions $\operatorname{Re}\left(z^{n+1}\right)$ and $\operatorname{Im}\left(z^{n+1}\right)$ are the intersections of the contours

$$
\frac{\partial \psi_{n+1}^{\mathrm{Re}}}{\partial x}=0 \quad \text { and } \quad \frac{\partial \psi_{n+1}^{\operatorname{Im}}}{\partial x}=0
$$

with

$$
\alpha_{n} \psi_{n+1}^{\operatorname{Re}}=\operatorname{Re}\left(z^{n+1}\right)-p_{n}, \quad \beta_{n} \psi_{n+1}^{\operatorname{Im}}=\operatorname{Im}\left(z^{n+1}\right)-q_{n}
$$

where

$$
\psi_{n+1}^{\mathrm{Re}}, \psi_{n+1}^{\mathrm{Im}} \in \Phi_{n+1}(\widehat{K}) ; \quad p_{n}, q_{n} \in V_{n}(\widehat{K}) .
$$

Proof. Clearly $P_{n+1}(\widehat{K}) \ni \operatorname{Re}\left(z^{n+1}\right), \operatorname{Im}\left(z^{n+1}\right) \notin V_{n}(\widehat{K})$. Since

$$
\begin{aligned}
P_{n+1}(\widehat{K}) & =P P_{n+1}(\widehat{K}) \cup S_{n}(\widehat{K}) \\
& =P P_{n+1}(\widehat{K}) \cup V_{n}(\widehat{K}) \\
& =\Psi_{n+1}(\widehat{K}) \cup V_{n}(\widehat{K}) \\
& =\Phi_{n+1}(\widehat{K}) \cup V_{n}(\widehat{K}) \\
& =\Phi_{n+1}(\widehat{K}) \oplus V_{n}(\widehat{K}),
\end{aligned}
$$

then

$$
\operatorname{Re}\left(z^{n+1}\right)=\alpha_{n} \psi_{n+1}^{\operatorname{Re}}+p_{n}, \quad \operatorname{Im}\left(z^{n+1}\right)=\beta_{n} \psi_{n+1}^{\operatorname{Im}}+q_{n}
$$


where

$$
\psi_{n+1}^{\mathrm{Re}}, \psi_{n+1}^{\mathrm{Im}} \in \Phi_{n+1}(\widehat{K}) ; \quad p_{n}, q_{n} \in V_{n}(\widehat{K}) .
$$

By Theorem 2.1, the conclusion follows.

\section{Derivative Superconvergent POINTS OF haRmonic FunCtions}

Denote $H_{n}(x)\left(H_{n}(y)\right)$ the set of derivative superconvergent points of the local finite element space $V_{n}(\widehat{K})$ for harmonic functions in the $x$-direction ( $y$-direction). Then according to Theorem 2.2,

$$
\begin{aligned}
& H_{n}(x)=\left\{(x, y) \in \widehat{K} \mid \quad \frac{\partial \psi_{n+1}^{\mathrm{Re}}}{\partial x}(x, y)=0, \quad \frac{\partial \psi_{n+1}^{\mathrm{Im}}}{\partial x}(x, y)=0\right\} \\
& H_{n}(y)=\left\{(x, y) \in \widehat{K} \mid \quad \frac{\partial \psi_{n+1}^{\mathrm{Re}}}{\partial y}(x, y)=0, \quad \frac{\partial \psi_{n+1}^{\mathrm{Im}}}{\partial y}(x, y)=0\right\}
\end{aligned}
$$

Case 1. $P_{n+1}(\widehat{K}) \backslash\left\{x^{n+1}, y^{n+1}\right\} \subset V_{n}(\widehat{K}) \subset Q_{n}(\widehat{K})$. This includes the intermediate family, tensor-product space, and all possible choices in between.

$$
\Phi_{n+1}(\widehat{K})=\operatorname{Span}\left\{\phi_{n+1}(x), \phi_{n+1}(y)\right\} .
$$

Therefore, both $\psi_{n+1}^{\operatorname{Re}}(x, y)$ and $\psi_{n+1}^{\operatorname{Im}}(x, y)$ are linear combinations of $\phi_{n+1}(x)$ and $\phi_{n+1}(y)$, and hence

$$
\begin{aligned}
& H_{n}(x)=\left\{(x, y) \in \widehat{K} \mid \quad \frac{\partial \phi_{n+1}}{\partial x}(x)=0\right\}=\left\{\left(G_{i}^{(n)}, y\right), \quad i=1, \ldots, n\right\} \\
& H_{n}(y)=\left\{(x, y) \in \widehat{K} \mid \quad \frac{\partial \phi_{n+1}}{\partial y}(y)=0\right\}=\left\{\left(x, G_{i}^{(n)}\right), \quad i=1, \ldots, n\right\} .
\end{aligned}
$$

Here $G_{i}^{(n)}$ are zeros of the Legendre polynomial $L_{n}$, i.e., the Gaussian points of degree $n$. In this case, superconvergent points are the same as those of the Poisson equation. Especially, in case of the intermediate and the tensor-product elements, we have confirmed the computer findings of [2].

Case 2. $V_{n}(\widehat{K})=S_{n}(\widehat{K})$, the serendipity family. In this case,

$$
\Phi_{n+1}(\widehat{K})=\Psi_{n+1}(\widehat{K}) \backslash V_{n}(\widehat{K})=\Psi_{n+1}(\widehat{K}) .
$$

Based on Theorem 2.2, the following procedure is developed to find the desired superconvergent points. Some properties and particular expressions of the Legendre polynomials are needed for this purpose. These properties and expressions are listed in the Appendix for readers' convenience. For more information regarding the Legendre polynomials, see [4].

Step 1. Orthogonal decomposition of the periodic polynomials. The following is a list of $\Psi_{n+1}(\widehat{K})$ for $n=1,2,3,4,5,6$. Note that for $n \leq 2$, the serendipity element 
$S_{n}(\widehat{K})$ is the same as the intermediate element.

$$
\begin{aligned}
& \Psi_{2}(\widehat{K})=\operatorname{Span}\left\{\phi_{2}(x), \phi_{2}(y)\right\} \\
& \Psi_{3}(\widehat{K})=\operatorname{Span}\left\{\phi_{3}(x), \phi_{3}(y)\right\} \\
& \Psi_{4}(\widehat{K})=\operatorname{Span}\left\{\phi_{4}(x), L_{2}(x) L_{2}(y), \phi_{4}(y)\right\} \\
& \Psi_{5}(\widehat{K})=\operatorname{Span}\left\{\phi_{5}(x), \phi_{3}(x) L_{2}(y), L_{2}(x) \phi_{3}(y), \phi_{5}(y)\right\} \\
& \Psi_{6}(\widehat{K})= \operatorname{Span}\left\{\phi_{6}(x),\left(L_{4}(x)-\frac{1}{2} L_{2}(x)\right) L_{2}(y), \phi_{3}(x) \phi_{3}(y),\right. \\
&\left.L_{2}(x)\left(L_{4}(y)-\frac{1}{2} L_{2}(y)\right), \phi_{6}(y)\right\}, \\
& \Psi_{7}(\widehat{K})=\operatorname{Span}\left\{\phi_{7}(x),\left(\phi_{5}(x)-\beta \phi_{3}(x)\right) L_{2}(y),\left(L_{4}(x)-\gamma L_{2}(x)\right) \phi_{3}(y),\right. \\
&\left.\phi_{3}(x)\left(L_{4}(y)-\gamma L_{2}(y)\right), L_{2}(x)\left(\phi_{5}(y)-\beta \phi_{3}(y)\right), \phi_{7}(y)\right\},
\end{aligned}
$$

where

$$
\beta=\frac{\left(\phi_{5}, \phi_{3}\right)\left\|L_{2}^{\prime}\right\|^{2}}{\left\|L_{2}\right\|^{4}+\left\|\phi_{3}\right\|^{2}\left\|L_{2}^{\prime}\right\|^{2}}, \quad \gamma=\frac{\left(L_{4}^{\prime}, L_{2}^{\prime}\right)\left\|\phi_{3}\right\|^{2}}{\left\|L_{2}\right\|^{4}+\left\|\phi_{3}\right\|^{2}\left\|L_{2}^{\prime}\right\|^{2}} .
$$

Step 2. Expression of harmonic functions by the orthogonal periodic polynomials of degree $n+1$ and the serendipity element of degree $n$.

$$
\begin{gathered}
\operatorname{Re}\left(z^{2}\right)=x^{2}-y^{2}=2 \sqrt{\frac{2}{3}}\left(\phi_{2}(x)-\phi_{2}(y)\right), \\
\operatorname{Re}\left(z^{3}\right)=x^{3}-3 x y^{2}=2 \sqrt{\frac{2}{5}} \phi_{3}(x)+p_{2}, \\
\operatorname{Re}\left(z^{4}\right)=x^{4}-6 x^{2} y^{2}+y^{4}=\frac{8}{5} \sqrt{\frac{2}{7}}\left(\phi_{4}(x)+\phi_{4}(y)\right)-\frac{8}{3} L_{2}(x) L_{2}(y)+p_{3}, \\
\operatorname{Re}\left(z^{5}\right)=x^{5}-10 x^{3} y^{2}+5 x y^{4}=\frac{8}{3} \sqrt{2}\left[\frac{1}{7} \phi_{5}(x)-\sqrt{5} \phi_{3}(x) L_{2}(y)\right]+p_{4}, \\
\operatorname{Re}\left(z^{6}\right)=x^{6}-15 x^{4} y^{2}+15 x^{2} y^{4}-y^{6}=\frac{16}{7}\left[\frac{1}{3} \sqrt{\frac{2}{11}}\left(\phi_{6}(x)-\phi_{6}(y)\right)\right. \\
\left.-\left(L_{4}(x)-\frac{1}{2} L_{2}(x)\right) L_{2}(y)+L_{2}(x)\left(L_{4}(y)-\frac{1}{2} L_{2}(y)\right)\right]+p_{5}, \\
\operatorname{Im}\left(z^{6}\right)=6 x^{5} y-20 x^{3} y^{3}+6 x y^{5}=-32 \phi_{3}(x) \phi_{3}(y)+q_{5} .
\end{gathered}
$$

Here $p_{r}, q_{r} \in S_{r}(\widehat{K})$. Note that

$$
x^{r} y, x y^{r} \in S_{r}(\widehat{K}), \quad \operatorname{Im}\left(z^{2}\right) \in S_{1}(\widehat{K}), \quad \operatorname{Im}\left(z^{4}\right) \in S_{3}(\widehat{K}) .
$$

Also note that $\operatorname{Im}\left(z^{3}\right), \operatorname{Im}\left(z^{5}\right)$ can be obtained symmetrically.

Step 3. Obtaining $\psi_{n+1}^{\mathrm{Re}}$ and $\psi_{n+1}^{\mathrm{Im}}$.

$$
\begin{aligned}
&\left\{\psi_{2}^{\mathrm{Re}}(x, y), \psi_{2}^{\operatorname{Im}}(x, y)\right\}=\left\{\phi_{2}(x)-\phi_{2}(y), 0\right\}, \\
&\left\{\psi_{3}^{\mathrm{Re}}(x, y), \psi_{3}^{\operatorname{Im}}(x, y)\right\}=\left\{\phi_{3}(x), \phi_{3}(y)\right\}, \\
&\left\{\psi_{4}^{\mathrm{Re}}(x, y), \psi_{4}^{\operatorname{Im}}(x, y)\right\}=\left\{\frac{1}{5} \sqrt{\frac{2}{7}}\left(\phi_{4}(x)+\phi_{4}(y)\right)-\frac{1}{3} L_{2}(x) L_{2}(y), 0\right\}, \\
&\left\{\psi_{5}^{\mathrm{Re}}(x, y), \psi_{5}^{\operatorname{Im}}(x, y)\right\}=\left\{\frac{1}{7} \phi_{5}(x)-\sqrt{5} \phi_{3}(x) L_{2}(y), \frac{1}{7} \phi_{5}(y)-\sqrt{5} \phi_{3}(y) L_{2}(x)\right\},
\end{aligned}
$$




$$
\begin{aligned}
\left\{\psi_{6}^{\mathrm{Re}}(x, y), \psi_{6}^{\mathrm{Im}}(x, y)\right\}=\left\{\frac{1}{3} \sqrt{\frac{2}{11}}\left(\phi_{6}(x)-\phi_{6}(y)\right)-\left(L_{4}(x)-\frac{1}{2} L_{2}(x)\right) L_{2}(y)\right. \\
\left.+L_{2}(x)\left(L_{4}(y)-\frac{1}{2} L_{2}(y)\right), \phi_{3}(x) \phi_{3}(y)\right\} .
\end{aligned}
$$

Step 4. Calculating $x$-derivative superconvergent points.

4.1. It is easy to see that the superconvergent points for $S_{1}(\widehat{K})$ and $S_{2}(\widehat{K})$ are along the lines of $L_{1}(x)=0$ and $L_{2}(x)=0$, respectively. They are the same as for the Poisson equation.

4.2. Superconvergent points for $S_{3}(\widehat{K})$ located on the contour of

$$
\frac{\partial}{\partial x}\left[\frac{1}{5} \sqrt{\frac{2}{7}}\left(\phi_{4}(x)+\phi_{4}(y)\right)-\frac{1}{3} L_{2}(x) L_{2}(y)\right]=\frac{1}{5} L_{3}(x)-x L_{2}(y)=0,
$$

or

$$
x\left(x^{2}-\frac{3}{5}\right)=x\left(3 y^{2}-1\right) .
$$

They are three curves in the reference element $\widehat{K}=[-1,1]^{2}: \quad x=0$ and two branches of the hyperbola $15 y^{2}-5 x^{2}=2$. Note that the four intersections of the hyperbola with $L_{3}(x)=0$

$$
\left(-\sqrt{\frac{3}{5}},-\frac{1}{\sqrt{3}}\right), \quad\left(\sqrt{\frac{3}{5}},-\frac{1}{\sqrt{3}}\right), \quad\left(\sqrt{\frac{3}{5}}, \frac{1}{\sqrt{3}}\right), \quad\left(-\sqrt{\frac{3}{5}}, \frac{1}{\sqrt{3}}\right),
$$

and the segment $(0, y),-1 \leq y \leq 1$, are also superconvergent points for the Poisson equation.

4.3. Superconvergent points for $S_{4}(\widehat{K})$ located at the intersections of contours

$$
\frac{\partial}{\partial x}\left[\frac{1}{7} \phi_{5}(x)-\sqrt{5} \phi_{3}(x) L_{2}(y)\right]=\frac{1}{\sqrt{2}}\left[\frac{3}{7} L_{4}(x)-5 L_{2}(x) L_{2}(y)\right]=0
$$

and

$$
\frac{\partial}{\partial x}\left[\frac{1}{7} \phi_{5}(y)-\sqrt{5} \phi_{3}(y) L_{2}(x)\right]=-3 \sqrt{5} \phi_{3}(y) x=0 .
$$

Using $L_{2}(0)=-1 / 2, L_{4}(0)=3 / 8$, and $L_{2}( \pm 1)=1$, we obtain three groups of superconvergent points.

4.3.1. $x=0$ and $y$ satisfies

$$
y^{2}=\frac{1}{3}-\frac{3}{70}
$$

4.3.2. $y=0$ and $x$ satisfies

$$
\frac{3}{7} L_{4}(x)+\frac{5}{2} L_{2}(x)=0 \quad \text { or } \quad 105 x^{4}+120 x^{2}-61=0 .
$$

There are two real roots in $[-1,1]$ :

$$
x^{2}=-\frac{4}{7}+\frac{1}{105} \sqrt{60^{2}+105 \cdot 61}=\sqrt{\frac{4^{2}}{7^{2}}+\frac{61}{105}}-\frac{4}{7} .
$$


4.3.3. $y= \pm 1$ and $x$ satisfies

$$
\frac{3}{7} L_{4}(x)-5 L_{2}(x)=0 \quad \text { or } \quad 105 x^{4}-510 x^{2}+149=0 .
$$

There are two real roots in $[-1,1]$ :

$$
x^{2}=\frac{51}{21}-\frac{1}{105} \sqrt{255^{2}-105 \cdot 149}=\frac{51}{21}-\sqrt{\frac{17^{2}}{7^{2}}-\frac{149}{105}} .
$$

4.4. Superconvergent points of $S_{5}(\widehat{K})$ located at the intersections of contours

$$
\frac{1}{3} L_{5}(x)-\left(L_{4}^{\prime}(x)-\frac{1}{2} L_{2}^{\prime}(x)\right) L_{2}(y)+L_{2}^{\prime}(x)\left(L_{4}(y)-\frac{1}{2} L_{2}^{\prime}(y)\right)
$$

and

$$
L_{2}(x) \phi_{3}(y)=0 .
$$

Again, we have three groups of points.

4.4.1. $y=0$ and $x$ satisfies

$$
\frac{1}{3} L_{5}(x)+\frac{1}{2}\left(L_{4}^{\prime}(x)-\frac{1}{2} L_{2}^{\prime}(x)\right)+\frac{5}{8} L_{2}^{\prime}(x)=0,
$$

which is

$$
x\left(\frac{21}{8} x^{4}+\frac{35}{6} x^{2}-2\right)=0 .
$$

There are three real roots in $[-1,1]$ :

$$
x=0, \quad x^{2}=-\frac{10}{9}+\frac{4}{21} \sqrt{\frac{35^{2}}{6^{2}}+21}=-\frac{10}{9}+\frac{4}{3} \sqrt{\frac{25}{36}+\frac{3}{7}} .
$$

4.4.2. $y= \pm 1$ and $x$ satisfies (note that $L_{2 k}( \pm 1)=1$ )

$$
\frac{1}{3} L_{5}(x)-\left(L_{4}^{\prime}(x)-\frac{1}{2} L_{2}^{\prime}(x)\right)+\frac{1}{2} L_{2}^{\prime}(x)=0,
$$

which is

$$
x\left(21 x^{4}-\frac{490}{3} x^{2}+89\right)=0 .
$$

There are three real roots in $[-1,1]$ :

$$
x=0, \quad x^{2}=\frac{35}{9}-\frac{1}{21} \sqrt{\frac{245^{2}}{3^{2}}-21 \cdot 89}=\frac{35}{9}-\sqrt{\frac{35^{2}}{9^{2}}-\frac{89}{21}} .
$$

4.4.3. $x= \pm \frac{1}{\sqrt{3}}$ and $y$ satisfies

$$
\mp \frac{\sqrt{3}}{54}-\left(\mp \frac{5}{9} \sqrt{3} \mp \frac{\sqrt{3}}{2}\right) L_{2}(y) \pm \sqrt{3}\left(L_{4}(y)-\frac{1}{2} L_{2}(y)\right)=0 .
$$

Note that

$$
L_{2}^{\prime}\left( \pm \frac{1}{\sqrt{3}}\right)= \pm \sqrt{3}, \quad L_{4}^{\prime}\left( \pm \frac{1}{\sqrt{3}}\right)=\mp \frac{5}{9} \sqrt{3}, \quad L_{5}\left( \pm \frac{1}{\sqrt{3}}\right)=\mp \frac{\sqrt{3}}{18} .
$$

The above equation is simplified to

$$
L_{4}(y)+\frac{5}{9} L_{2}(y)-\frac{1}{54}=0 .
$$


TABLE 1. Superconvergent points $(x, y)$ of harmonic functions $n=4$.

$$
\begin{array}{rl}
0.00000000000000 & 0.53895843112080 \\
0.00000000000000 & -0.53895843112080 \\
0.61740622481152 & 0.00000000000000 \\
-0.61740622481152 & 0.00000000000000 \\
0.55877322236109 & 1.00000000000000 \\
-0.55877322236109 & 1.00000000000000 \\
0.55877322236109 & -1.00000000000000 \\
-0.55877322236109 & -1.00000000000000 \\
\sqrt{\frac{1}{3}-\frac{3}{70}} & =0.53895843112080 \cdots \\
\sqrt{\frac{4^{2}}{7^{2}}+\frac{61}{105}-\frac{4}{7}} & =0.61740622481152 \cdots \\
\sqrt{\frac{51}{21}-\sqrt{\frac{17^{2}}{7^{2}}-\frac{149}{105}}} & =0.55877322236109 \cdots
\end{array}
$$

There are four real roots in $[-1,1]$ :

$$
y^{2}=\frac{1}{3}\left(1 \pm \sqrt{1-\frac{17}{105}}\right) .
$$

Summarizing the results for $n=4$ and $n=5$, we list in Tables 1 and 2, 14 digits of $x$ and $y$ coordinates of superconvergent points.

Comparing with the data provided in [2], it is interesting to observe that all 10 digits of computer findings are correct (up to rounding at the tenth digits) with one exception: instead of 0.1678536898 , [2] listed 0.1678536900 .

The superconvergent points for the $y$-derivative can be obtained similarly. Summing up, we conclude that:

1. For any finite element space that is contained in the tensor-product space and contains the intermediate element, all superconvergent points for harmonic functions under the rectangular mesh are along Gaussian lines, the same as those for the Poisson equation.

2. For the serendipity element of order $n=3$, the superconvergent points are along the central line $x=0$ and the two branches of the hyperbola $15 y^{2}-5 x^{2}=2$.

3 . For the serendipity element of order $n=4$, there are eight superconvergent points for harmonic functions compared to none for the Poisson equation; and for the serendipity element of order $n=5$, there are 17 superconvergent points for harmonic functions compared to three symmetry points for the Poisson equation.

Remark 4.1. Results in the computer-based proof of [2] for harmonic function under the rectangular mesh are justified for $n=1,2,3,4,5$. However, the results here are more general in the sense that they include all possible choices of the finite element space between the intermediate family and the tensor-product space.

Remark 4.2. Reference [2] also listed data for $n=6,7$. We did not go further since it would be all technical. In general, for $n=2 k$ or $n=2 k+1$, root-finding of a polynomial of degree $k$ needs to be performed to locate the desired superconvergent points. 
TABLE 2. Superconvergent points $(x, y)$ of harmonic functions $n=5$.

$\begin{array}{rr}0.00000000000000 & 0.00000000000000 \\ 0.54941314054283 & 0.00000000000000 \\ -0.54941314054283 & 0.00000000000000 \\ 0.00000000000000 & 1.00000000000000 \\ 0.76784878647334 & 1.00000000000000 \\ -0.76784878647334 & 1.00000000000000 \\ 0.00000000000000 & -1.00000000000000 \\ 0.76784878647334 & -1.00000000000000 \\ -0.76784878647334 & -1.00000000000000 \\ 0.57735026918963 & 0.79905682243383 \\ 0.57735026918963 & -0.79905682243383 \\ 0.57735026918963 & 0.16785368982726 \\ 0.57735026918963 & -0.16785368982726 \\ -0.57735026918963 & 0.79905682243383 \\ -0.57735026918963 & -0.79905682243383 \\ -0.57735026918963 & 0.16785368982726 \\ -0.57735026918963 & -0.16785368982726\end{array}$

$$
\begin{aligned}
\sqrt{\frac{4}{3} \sqrt{\frac{5^{2}}{6^{2}}+\frac{3}{7}-\frac{10}{9}}} & =0.54941314054283 \cdots \\
\sqrt{\frac{35}{9}-\sqrt{\frac{35^{2}}{9^{2}}-\frac{89}{21}}} & =0.76784878647334 \cdots \\
\frac{1}{\sqrt{3}} \sqrt{1+\sqrt{1-\frac{17}{105}}} & =0.79905682243383 \cdots \\
\frac{1}{\sqrt{3}} \sqrt{1-\sqrt{1-\frac{17}{105}}} & =0.16785368982726 \cdots \\
\frac{1}{\sqrt{3}} & =0.57735026918963 \cdots
\end{aligned}
$$

Appendix. LeGENDRE POLYNOMials

$$
\begin{gathered}
L_{0}(x)=1, \quad L_{1}(x)=x, \quad(k+1) L_{k+1}(x)=(2 k+1) x L_{k}(x)-k L_{k-1}(x) . \\
2 L_{2}(x)=3 x^{2}-1, \quad 2 L_{3}(x)=5 x^{3}-3 x, \\
8 L_{4}(x)=35 x^{4}-30 x^{2}+3, \quad 8 L_{5}(x)=63 x^{5}-70 x^{3}+15 x, \\
16 L_{6}(x)=231 x^{6}-315 x^{4}+105 x^{2}-5, \\
16 L_{7}(x)=429 x^{7}-693 x^{5}+315 x^{3}-35 x .
\end{gathered}
$$




$$
\begin{gathered}
\phi_{j+1}(x)=\sqrt{\frac{2 j+1}{2}} \int_{-1}^{x} L_{j}(t) d t=\frac{1}{\sqrt{2(2 j+1)}}\left[L_{j+1}(x)-L_{j-1}(x)\right] . \\
\phi_{2}(x)=\sqrt{\frac{3}{2}} \frac{1}{2}\left(x^{2}-1\right), \quad \phi_{3}(x)=\sqrt{\frac{5}{2}} \frac{1}{2}\left(x^{3}-x\right), \\
\phi_{4}(x)=\sqrt{\frac{7}{2}} \frac{1}{8}\left(5 x^{4}-6 x^{2}+1\right), \\
\phi_{5}(x)=\sqrt{\frac{9}{2}} \frac{1}{8}\left(7 x^{5}-10 x^{3}+3 x\right), \\
\phi_{6}(x)=\sqrt{\frac{11}{2}} \frac{1}{16}\left(21 x^{6}-35 x^{4}+15 x^{2}-1\right), \\
\phi_{7}(x)=\sqrt{\frac{13}{2}} \frac{1}{16}\left(33 x^{7}-63 x^{5}+35 x^{3}-5 x\right), \\
\phi_{8}(x)=\sqrt{\frac{15}{2}} \frac{1}{128}\left(429 x^{8}-924 x^{6}+630 x^{4}-140 x^{2}+5\right) .
\end{gathered}
$$

\section{ACKNOWLEDGMENT}

This research is partially supported by the National Science Foundation grants DMS-0074301, DMS-0079743, and INT-0196139.

\section{REFERENCES}

1. I. Babuška and T. Strouboulis, The Finite Element Method and its Reliability, Oxford University Press, London, 2001.

2. I. Babuška, T. Strouboulis, C.S. Upadhyay, and S.K. Gangaraj, Computer-based proof of the existence of superconvergence points in the finite element method; superconvergence of the derivatives in finite element solutions of Laplace's, Poisson's, and the elasticity equations, Numer. Methods Partial Differential Equations 12 (1996), 347-392. MR 97c:65160

3. M. Kř́̌žek, P. Neittaanmäki, and R. Stenberg (Eds.), Finite element methods. Superconvergence, post-processing, and a posteriori estimates, Lecture Notes in Pure and Applied Mathematics Series, Vol. 196, Marcel Dekker, New York, 1997. MR 98i:65003

4. N.N. Lebedev, Special functions and their applications, Dover, New York, 1972. MR 50:2568

5. A.H. Schatz, I.H. Sloan, and L.B. Wahlbin, Superconvergence in finite element methods and meshes which are symmetric with respect to a point, SIAM J. Numer. Anal. 33(2) (1996), 505-521. MR 98f:65112

6. L.B. Wahlbin, Superconvergence in Galerkin finite element methods, Lecture Notes in Mathematics, Vol. 1605, Springer, Berlin, 1995. MR 98j:65083

7. Zhimin Zhang, Derivative superconvergent points in finite element solutions of Poisson's equation for the serendipity and intermediate families - A theoretical justification, Math. Comp. 67 (1998), 541-552. MR 98i:65104

Department of Mathematics, Wayne State University, Detroit, Michigan 48202

E-mail address: zzhang@math.wayne.edu 